\title{
Singular Inflation
}

\author{
John D. Barrow* and Alexander A. H. Graham ${ }^{\dagger}$ \\ Department of Applied Mathematics and Theoretical Physics \\ Centre for Mathematical Sciences, University of Cambridge, Wilberforce Road, CB3 OWA, UK
}

(Dated: August 26, 2021)

\begin{abstract}
We prove that a homogeneous and isotropic universe containing a scalar field with a power-law potential, $V(\phi)=A \phi^{n}$, with $0<n<1$ and $A>0$ always develops a finite-time singularity at which the Hubble rate and its first derivative are finite, but its second derivative diverges. These are the first examples of cosmological models with realistic matter sources that possess weak singularities of 'sudden' type. We also show that a large class of models with even weaker singularities exist for noninteger $n>1$. More precisely, if $k<n<k+1$ where $k$ is a positive integer then the first divergence of the Hubble rate occurs with its $(k+2)$ th derivative. At early times these models behave like standard large-field inflation models but they encounter a singular end-state when inflation ends. We term this singular inflation.
\end{abstract}

PACS numbers: 98.80.Bp, 98.80.Cq, 98.80.Es, 98.80.Jk

\section{INTRODUCTION}

When can a universe possess a future singularity? [1] In the absence of quantum effects, the best known case where this can happen, first found by Friedmann [2], is a future 'big crunch' singularity of infinite density. It is essentially the time reversal of the big bang singularity: the universe expands to a maximum size at some time, contracts, and then collapses to the future singularity. This occurs in an isotropic universe if the cosmological constant, $\Lambda$, is negative, or if the universe is positively curved and contains a perfect fluid with bounded pressure obeying the strong energy condition.

A much more drastic type of future singularity is the 'big rip'. This was first studied in Refs. [3-5]. It occurs when the universe contains a fluid violating the null energy condition. The expansion is so rapid that the expansion scale factor and all its time-derivatives diverge in finite time. For a perfect fluid with equation of state $p=w \rho$, linking its pressure $p$ and density $\rho$, a 'big rip' occurs when $w<-1$.

By contrast, a far weaker type of finite-time singularity is a 'sudden singularity'. Strikingly, it does not require cosmological contraction to occur. These singularities were first discovered in Ref. [6] as a counterexample to the belief (Ref. [7]) that compliance with the strong and weak energy conditions and the positive pressure criterion alone suffice to ensure that a spatially closed universe with $S^{3}$ topology has a maximal hypersurface and recollapses. They were later discussed systematically in Refs. [8, 9] (see Refs. [10, 11] for similar singularities in brane-world models). They are characterised by the scale factor, $a(t)$, and its first derivative $\dot{a}(t)$ being finite as $t \rightarrow t_{s}$, but $\ddot{a}(t) \rightarrow \infty$ as $t \rightarrow t_{s}$. At these singularities $\rho$ is finite but $p \rightarrow+\infty$, and $\rho+3 p>0$. Generalised sudden singularities can also be constructed where the

\footnotetext{
* J.D.Barrow@damtp.cam.ac.uk

$\dagger$ A.A.H.Graham@damtp.cam.ac.uk
}

singularity occurs in arbitrarily high derivatives of $a(t)$, with all lower derivatives finite [9]. For further studies of these classical singularities see Refs. [12-18] and for discussions of quantum effects see Refs. [19, 20].

Sudden singularities stand in stark contrast to big rip singularities as they are much weaker. Firstly, they satisfy all of the classical energy conditions bar the dominant energy condition (and generalised sudden singularities satisfy them all) [21]. Secondly, while they are scalar polynomial curvature singularities, they are 'weak' in the sense of both Tipler [22] and Krolak [23] (this means they are not "crushing" singularities because an object approaching the singularity is not crushed to zero volume). Moreover, geodesics are actually extendible through sudden singularities [24], unlike for big rip and big crunch singularities ${ }^{1}$, and this behaviour is stable [25]. For other types of cosmological singularities discussed in the literature see Refs. [26-31].

Unfortunately, unlike the known examples of a big rip singularity, we do not have a simple physically wellmotivated matter model which produces a sudden (or generalised sudden) singularity. Most studies of these singularities postulate a form of the scale factor with the desired singularity and then use the Einstein equations to find energy-condition compliant $\rho$ and $p$ behaviours which source this solution - although a solution of this sort is general in the function-counting sense [14].

Here, we will construct for the first time a large family of finite-time cosmological singularities for a canonical scalar field with a simple, power-law potential. We show that the formation of these singularities is a general feature of these simple matter models. They provide the

\footnotetext{
1 Note in this paper the term 'singularity' is used somewhat loosely to denote a point where some, in principle, observable quantity (such as $H$ or its higher order derivatives) becomes unbounded at finite time. It is not necessarily accompanied by geodesic incompleteness, and thereby the Hawking-Penrose singularity theorems do not apply in this case. The somewhat informal terminology is common in the literature in this subject; a better term for these very weak singularities might be singular events.
} 
first examples of cosmological models with realistic matter which evolve towards weak, finite-time singularities. They also have the interesting feature that, for appropriate initial conditions, they can describe large-field inflation in the early universe. The qualitative difference to standard inflation models is that when inflation ends they evolve to a singular state in finite time.

\section{SCALAR FIELD COSMOLOGIES}

We consider a spatially-flat Friedmann-LemaîtreRobertson-Walker (FLRW) universe, with scale factor $a(t)$ and Hubble parameter $H(t)=\dot{a}(t) / a(t)$. We assume the universe contains only a scalar field, $\phi(t)$, with selfinteraction potential $V(\phi)$. The Einstein and scalar field equations of motion are (in units where $c=8 \pi G=1$ )

$$
\begin{aligned}
& 3 H^{2}=\frac{1}{2} \dot{\phi}^{2}+V(\phi), \\
& \dot{H}=-\frac{1}{2} \dot{\phi}^{2}, \\
& \ddot{\phi}+3 H \dot{\phi}+V^{\prime}(\phi)=0,
\end{aligned}
$$

where $^{\prime}=d / d \phi$. We consider the case where the potential takes a power-law form:

$$
V(\phi)=A \phi^{n},
$$

where $A>0$ is a constant. When $n$ is a positive even integer it provides the classic example of a large-field inflation model with a single potential minimum. If the initial conditions are chosen so that the scalar field starts high enough up the potential then the universe inflates as the $\phi$ field rolls slowly down the potential. When it nears the potential minimum at $\phi=0$ inflation ends and the field oscillates about the minimum. In particular, the case where $n=4$ is the original model of chaotic inflation [32]. When $n$ is a positive odd integer the universe appears to recollapses under the influence of the scalar field (for the $n=1$ case see Ref. [33]). We will be interested in the case where $n>0$ and is not an integer.

\section{A. Finite-time singularities when $0<n<1$}

We first examine the case where $0<n<1$. Eq. (3) becomes

$$
\ddot{\phi}=-3 H \dot{\phi}-A n \phi^{n-1} .
$$

At $t=0$ we choose initial conditions so that $\phi_{0} \equiv \phi(0)>$ 0 (cases where $\phi_{0}<0$ are not cosmologically relevant, and generally unphysical), but the value of $\dot{\phi}_{0} \equiv \dot{\phi}(0)$ is unconstrained. We assume that the universe is expanding initially, so $H_{0} \equiv H(0)>0$. It is not difficult to see how the system evolves in time. If we start with $\dot{\phi}_{0}>0$ then both terms on the right-hand side of Eq. (5) are negative, so in a finite time $\dot{\phi}$ becomes negative. Hence, in finite time the scalar field starts to decrease, and since $\dot{\phi}$ continues to decrease, because the second term on the right-hand side of Eq. (5) increases as $\phi$ decreases, it will reach $\phi=0$ in finite time. When this happens $\ddot{\phi} \rightarrow-\infty$ (since $n-1<0$ ) as $\phi \rightarrow 0$. From Eqs. (1)-(2), we see $H$ and $\dot{H}$ are both finite at this point (provided that $\dot{\phi}<0$ is finite), but $\ddot{H}$ diverges as

$$
\ddot{H}=-\dot{\phi} \ddot{\phi} \rightarrow-\infty \text { as } \phi \rightarrow 0 .
$$

Before we demonstrate these claims rigorously we make a few points about the nature of the singularity encountered. Firstly, note it is not a scalar polynomial (sp) curvature singularity, as both $H$ and $\dot{H}$ are finite at this point. For a spatially-flat FLRW spacetime the Ricci scalar, $R$, may be written as

$$
R=6\left(2 H^{2}+\dot{H}\right),
$$

which is clearly finite as $\phi \rightarrow 0$. However, higher scalar derivatives of the curvature (like $\partial_{a} R \partial^{a} R$ or $\square R$ ) are not regular since

$$
\dot{R}=6(4 H \dot{H}+\ddot{H}) \rightarrow-\infty \text { as } \phi \rightarrow 0 .
$$

These are generalised sudden singularities and examples of what Ellis and Schmidt call $C^{k}$ scalar curvature singularities [34]. We might call them scalar differential singularities. They are the first examples of such weak singularities in an FLRW spacetime for a simple matter model (see Ref. [35] for similar singularities in tilted Bianchi spacetimes). Regularity of the curvature ensures that they are weak in the sense of both Tipler [22] and Krolak [23]. Furthermore, since they are weaker than sudden singularities the spacetime is geodesically complete and extendible at the finite-time singularities.

We can ask which of the classical energy conditions these singularities obey. A canonical scalar field is equivalent to a perfect fluid with density $\rho=\frac{1}{2} \dot{\phi}^{2}+V$ and pressure $p=\frac{1}{2} \dot{\phi}^{2}-V$. This implies that the null energy condition $(\rho+p \geq 0)$ is always satisfied for any choice of $V(\phi)$. Moreover, if $V(\phi) \geq 0$, as in our case, then both the weak energy condition $(\rho \geq 0$ and $\rho+p \geq 0)$ and the dominant energy condition $(\rho \geq 0$ and $\rho \geq|p|)$ are satisfied. The strong energy condition $(\rho+p \geq 0$ and $\rho+3 p \geq 0$ ) is satisfied if $\dot{\phi}^{2} \geq V$; while this initially may not hold depending on the initial conditions we choose (indeed, if this model is to function as inflation at early times it must be violated), it is always satisfied as $\phi \rightarrow 0$. Therefore all classical energy conditions are satisfied near the singularity. Again, this is the first example of this kind known for a simple matter model.

Let us now prove that the system does indeed develop a finite-time singularity of this type. It suffices to prove that the system reaches $\phi=0$ in finite time with $\dot{\phi}$ regular at this point and $\dot{\phi}<0$. To do so, we prove first that the system always reaches $\dot{\phi}=0$ in finite time, and then show that it must subsequently reach $\phi=0$ in finite time. Note that without loss of generality we may assume that 
$\dot{\phi}_{0}>0$, as otherwise we simply omit the first step in the argument.

To prove the first claim, note that in this region of parameter space $\ddot{\phi}<0$, so that $\dot{\phi}$ is strictly decreasing and so, while $\phi$ increases in this region, it cannot grow faster than linearly in time: $\phi(t) \leq \phi_{0}+\dot{\phi}_{0} t$. In Eq. (5) this implies that

$$
\ddot{\phi} \leq-n A \phi^{n-1} \leq-n A\left(\phi_{0}+\dot{\phi}_{0} t\right)^{n-1} .
$$

Integrating Eq. (9) once gives an upper bound on $\dot{\phi}$ :

$$
\dot{\phi}(t) \leq \dot{\phi}_{0}+\frac{A \phi_{0}^{n}}{\dot{\phi}_{0}}-\frac{A}{\dot{\phi}_{0}}\left(\phi_{0}+\dot{\phi}_{0} t\right)^{n} .
$$

This implies that $\dot{\phi}=0$ is reached in finite time. This completes the first part of the argument.

For the second part, we must show that the system will reach $\phi=0$ in finite time. Before we do so, first note that at the point where $\dot{\phi}=0$ we have $\ddot{\phi}=-A n \phi^{n-1}<0$, so there exists a later time, $T$, at which $\dot{\phi}(T)<0$, and $\dot{\phi}$ cannot become positive again. We will prove that $\phi=0$ is reached in finite time by showing that $\dot{\phi}$ is a decreasing function of time, by which we mean that $\dot{\phi}(t) \leq \dot{\phi}(T)$ for all $t>T$. Since $\dot{\phi}$ is negative this clearly suffices to show $\phi=0$ is reached, as integrating this inequality once gives $\phi(t) \leq \phi(T)+(t-T) \dot{\phi}(T)$, and so $\phi=0$ is reached within a time $t=\phi(T) /|\dot{\phi}(T)|$ from $t=T$.

To prove that $\dot{\phi}$ is decreasing, note that without loss of generality we may choose $T$ so that $\ddot{\phi}(T)<0$ as well. Now if $\dot{\phi}$ increases in the future there must exist a time $t_{1}>T$ at which $\dot{\phi}\left(t_{1}\right)=\dot{\phi}(T)$ and $\ddot{\phi}\left(t_{1}\right)>0$. However, this is impossible since Eq. (2) implies $H$ is a decreasing function of time and at this point Eq. (5) requires

$$
\begin{aligned}
& \ddot{\phi}\left(t_{1}\right)=-3 H\left(t_{1}\right) \dot{\phi}\left(t_{1}\right)-A n \phi\left(t_{1}\right)^{n-1} \\
& <-3 H(T) \dot{\phi}(T)-A n \phi(T)^{n-1}=\ddot{\phi}(T)<0,
\end{aligned}
$$

hence no such time exists and so $\dot{\phi}$ is always decreasing.

While we have proven that $\phi=0$ is always reached in a finite time, regardless of the initial conditions, we also need to show that $\dot{\phi}$ is regular at $\phi=0$. This is easily shown as $H(t)$ is a decreasing function of time and positive in this interval, so

$$
\dot{\phi}^{2}(\phi=0) \leq 2 A \phi^{n}(\dot{\phi}=0) .
$$

Since $\phi$ is finite at the turnover we conclude that $\dot{\phi}$ is finite at $\phi=0$, and as it is a decreasing function of time we must also have $\dot{\phi}<0$.

\section{B. Finite-time singularities when $n>1$}

Let us now examine the case where $n>1$ and $n$ is not an integer. Here, we also expect that $\phi=0$ should be reached in a finite time from generic initial data. In particular, it is possible to show that $\dot{\phi}=0$ is always reached in finite time, as from Eq. (5) we have that $\ddot{\phi}<-n A \phi_{0}^{n-1}$, which implies that

$$
\dot{\phi}(t) \leq \dot{\phi}_{0}-n A \phi_{0}^{n-1} t,
$$

so $\dot{\phi}=0$ is reached within time $t_{0}=\dot{\phi}_{0} / n A \phi_{0}^{n-1}$. Moreover, at this point $\ddot{\phi}<0$, so $\dot{\phi}$ becomes strictly negative and can never become positive, so $\phi$ always decreases from this point. It is not so easy to show analytically that $\phi=0$ is reached in finite time because the key step used in the argument when $0<n<1-$ that $\dot{\phi}$ is always a decreasing function - is no longer true when $n>1$. However, it is easy to see that the only alternative is that $\phi \rightarrow 0$ and $\dot{\phi} \rightarrow 0$ as $t \rightarrow \infty$. This situation is at most of measure zero, and it is easy to show via numerical simulations of Eqs. (1)-(3) that $\phi=0$ is indeed reached in finite time for generic initial conditions for any chosen non-integer value of $n>1$.

When $1<n<2$ we can show that $\phi=0$ is reached in finite time by the following argument. Firstly, without loss of generality we may assume that for $t>t_{1}>t_{0}$ we have $\ddot{\phi} \geq 0$, otherwise $\phi$ decreases even faster and so $\phi=0$ must be reached in finite time if it is reached when $\ddot{\phi} \geq 0$. Now $\ddot{\phi} \geq 0$ implies that for $t>t_{1}$ we have that

$$
\dot{\phi} \leq \frac{-A n \phi^{n-1}}{3 H} \leq \frac{-A n \phi^{n-1}}{3 H\left(t_{1}\right)}
$$

since $H(t)$ is a decreasing function of time. Integrating this once gives

$$
\phi^{2-n} \leq C-\frac{A n(2-n) t}{3 H\left(t_{1}\right)},
$$

where $C>0$ is a constant. Clearly then, if $n<2$ this implies that $\phi=0$ is reached in finite time.

When $n \geq 2$ one can use the results of Richard [36] to derive conditions under which $\phi=0$ is reached in finite time. Richard studied the existence of zeros of a class of second-order, non-linear ordinary differential equations which include Eq. (3). Applying his results to Eq. (3) we find a zero exists in finite time provided the function

$$
\theta(t)=-a(t)^{3} \frac{d}{d t}\left[\int_{0}^{t} a\left(t^{\prime}\right)^{3} d t^{\prime}\right]^{-\frac{2}{n+1}}
$$

is decreasing and $\theta \rightarrow 0$ as $t \rightarrow \infty$. Notice that in an expanding universe $\theta(t) \geq 0$, so in our case $\theta(t)$ always approaches zero from above. In general, $\theta(t)$ will be a decreasing function provided that $a(t)$ increases slowly enough. For instance, for a power-law expansion, $a(t) \propto$ $t^{m}$, then $\theta(t)$ is a decreasing function which tends to zero asymptotically provided that

$$
m<\frac{1}{3}+\frac{4}{3(n-1)} .
$$

That is, provided the expansion rate eventually approaches that of a kinetically-dominated solution the conditions are satisfied and $\phi=0$ is reached in finite time. 
When $\phi=0$ is reached Eq. (12) still implies $\dot{\phi}<0$ is finite. Moreover, Eq. (3) now implies that $\ddot{\phi}$ is finite at this point, unlike in the previous case. However, higher-order derivatives of $\phi$ diverge. For instance, differentiating Eq. (3) once gives that

$$
\dddot{\phi}-9 H^{2} \dot{\phi}-\frac{3}{2} \dot{\phi}^{3}-3 H V^{\prime}(\phi)+V^{\prime \prime}(\phi) \dot{\phi}=0 .
$$

For $1<n<2$, every term is finite as $\phi \rightarrow 0$ except the last term which is divergent, so $\dddot{\phi} \rightarrow \infty$ as $\phi \rightarrow 0$. This implies that the first divergence in the scale factor occurs at fourth order in its derivatives, since

$$
\dddot{H}=-\ddot{\phi}^{2}-\ddot{\phi} \ddot{\phi} \rightarrow \infty \text { as } \phi \rightarrow 0 .
$$

This implies, for instance, that $\square R$ and higher derivatives of the curvature are divergent on approach to this singularity.

It is not difficult to generalise these conclusions to arbitrarily large non-integer values of $n$. If $k<n<k+1$, where $k$ is a positive integer, then as $\phi \rightarrow 0$ we have $\phi^{(k+2)} \rightarrow(-1)^{k+1} \infty$, with all lower derivatives of $\phi$ finite. This implies that the first divergence of the Hubble rate occurs for the $(k+2)^{t h}$ derivative: $H^{(k+2)} \rightarrow(-1)^{k+1} \infty$ as $\phi \rightarrow 0$. If $n$ is an integer these singularities never occur as $V(\phi)$ is smooth at $\phi=0$.

\section{DISCUSSION}

We have shown that a power-law potential given by Eq. (4) admits an arbitrarily large family of ultra-weak generalised sudden singularities which satisfy all the classical energy conditions and are characterised by the divergence of a sufficiently high derivative of a curvature invariant. Polynomial curvature invariants are always finite upon approach to the singularity: the divergence always occurs in a derivative of a curvature invariant due to the non-analytic behaviour of the curvature at the singularity. More generally, we expect that any potential $V(\phi)$ which is not smooth at $\phi=0$ will admit singularities of a similar form.

It is important to note that these models describe inflationary cosmologies in the same way as large-field inflation models: we simply choose our initial conditions, $\phi_{0}$ and $\dot{\phi}_{0}$, so that the system starts high enough up the potential and is potential-dominated. Inflation then occurs while the field violates the strong energy condition, but it eventually ends as $\phi \rightarrow 0$ and the system enters the reheating phase. The only difference is when the system reaches $\phi=0$ deep in the reheating phase (using the slow-roll approximation this is reached in time $\left.t \approx \frac{2}{n(4-n)} \sqrt{\frac{3}{A}} \frac{\phi_{0}^{2-n / 2}}{M_{p}}\right)$. The difference is that reheating presumably proceeds differently. Since predictions for the power spectrum are insensitive to the behaviour at reheating, these models will give the same predictions for CMB observables as large-field inflation models. Inflation with fractional potentials has been studied before
[37], and can even be motivated from string theory [38], although their singular behaviour as $\phi \rightarrow 0$ had not been recognised. Note though that the arguments used to derive fractional potentials from string theoretic constructions are only valid for large field values in general. It is also worth noting that monomial potentials with $n<2$ given a better fit to current $\mathrm{CMB}$ data than large integer values do [39].

Let us briefly comment on the case when $n<0$ and $n$ is fractional. These potentials are more relevant as candidates for late-time quintessence than inflation. In this case, there is little difference between when $n$ is fractional and when it is an integer. One can easily show that if we start from initial data where $\phi_{0}>0$ then, generically, $\dot{\phi}$ becomes positive in finite time, and always remains positive from this point onwards. This is because in this case $\ddot{\phi}>0$ for non-zero $\phi$ at $\dot{\phi}=0$, so if $\phi$ is initially increasing it continues to do so forever. Even if we start from initial data where $\dot{\phi}_{0}<0$ then $\ddot{\phi}>0$ and so, by similar arguments to section II, $\dot{\phi}$ becomes positive in finite time. The scalar field therefore always increases at late times, and the evolution is non-singular towards the future.

To conclude, we have demonstrated the existence of a large family of new ultra-weak generalised sudden singularities in a spatially-flat FLRW universe with a scalar field possessing a simple power-law potential. They are the first examples of a spacetime possessing such weak singularities for a simple and realistic matter model. Moreover, their formation is completely generic in FLRW models (indeed, when $0<n<1$ they form from any isotropic and homogeneous initial data).

The singularities discovered here are generalised sudden singularities: the divergence in the scale factor occurs at no lower order than the third derivative, $\dddot{a}$, (as in Ref. [9]). Note that while we can find potentials leading to these very weak singularities, and find potentials in the same family which admit big crunch singularities, it is not so easy to construct scalar-field models which admit sudden singularities with divergence in $\ddot{a}$. It is not difficult to see why. At such a singularity we would require $H$ to be finite, while $\dot{H}$ diverges. This implies $\dot{\phi} \rightarrow \infty$ on approach to the singularity, and so $V(\phi)$ must diverge in such a way as to cancel precisely the divergence of $\dot{\phi}$. Such potentials will likely be unphysical, and if they do admit sudden singularities then the sensitive cancellation required will likely render them a set of measure zero. Sudden finite-time singularities with $\ddot{a} \rightarrow \infty$ are in some sense a borderline case between singularities which are definitely inextendible (big crunch or rip) and those which are not, so we could view our results as indicating that they may not exist in general for 'reasonable' forms of matter, but their generalised forms with $d^{n} a / d t^{n} \rightarrow \infty$ for $n \geq 3$ are common.

Although we restricted to spatially flat universes our arguments are unaffected if the universe is open. We also expect finite-time singularities to form in a closed universe provided they form before the universe reaches the expansion maximum, as in Ref. [6]. However, a mas- 
sive scalar field $(n=2)$ in a closed FLRW universe can have quite intricate fractal behaviour that allows a subset of initial data to avoid a strong curvature collapse singularity [40], so we might expect analogously complicated behaviour for non-integer potentials.

Note that we expect these singularities to be classically stable. This is because the formation of these singularities is stable within the family of FRLW spacetimes, so they can only be destabilised by the formation of large anisotropies or inhomogeneities as $\phi \rightarrow 0$. One can show they also generically form in the anisotropic case, and since sudden singularities are known to be classically stable [13] we expect these even weaker singularities to be likewise stable. Indeed, as sudden singularities have been shown to be stable [14] in the sense that the homogeneous solution is the leading order solution of the field equations containing nine independently arbitrary functions in the neighbourhood of the singularity we would expect these finite-time scalar-field singularities to be stable in this sense (however, the general scalar-field solution only contains six arbitrary spatial functions on a time slice). They are also probably even stable to quantum corrections, since the curvature on approach to the singularity is finite and we can construct singularities in which arbitrarily high derivatives of the curvature are finite [19, 20]. This is quite unlike most other examples of exotic singularities known.

A pressing question raised by this paper is what is the future evolution of these spacetimes beyond the point $\phi=$ 0 ? Since all polynomial curvature invariants are finite, the spacetime is extendible past this point. Notice that as $\phi \rightarrow 0$ (with $\dot{\phi}<0$ ) then the scale factor approaches that of a universe dominated by a stiff fluid: $a(t) \propto t^{\frac{1}{3}}$. The precise form of $\phi(t)$ on approach to the singular point is in general quite complicated and depends upon the precise form of $V(\phi)$. The problem with evolving the system beyond $\phi=0$ is that in some cases (for instance $n=\frac{1}{2}$ ) the matter model breaks down beyond the point $\phi=0$, since the naive evolution would push $\phi$ to become strictly negative, which in general would lead to the expansion rate becoming complex. Note though that this is only the case for some choices of $n$, and there are many choices (e.g. $n=\frac{1}{3}$ ) for which $V(\phi)$ is always real. In this case numerical evidence suggests that if $V(\phi)$ is negativedefinite for $\phi<0$ the spacetime collapses to a big crunch singularity, while if $V(\phi)$ is positive-definite for $\phi<0$ no such collapse occurs. Instead the universe goes through $\phi=0$ an infinite number of times. We hope to return to this issue in future work. Some ideas for how to approach this problem using distributional quantities are discussed in Refs. [41, 42].

A further question is how this model of inflation could be distinguished from a similar large-field model? Also, would these singularities reported here survive the detailed physics of reheating, which usually involves coupling the inflaton to one or more particles? This is of course impossible to answer without a specific model of reheating, but there is no reason to think reheating would alter them. One way to see this is that reheating can usually be modeled by adding a friction-like term, $\Gamma \dot{\phi}$, into Eq. (3), and it is not difficult to see this should not generally affect the ability of the field to reach $\phi=0$ [43]. The prospects of detecting an observable signature of this behaviour face the same challenges as connecting the details of reheating to CMB observations.

\section{ACKNOWLEDGEMENTS}

A.A.H.G. and J.D.B. are supported by the STFC. We thank Rahul Jha, Mary Graham, Stephen Siklos and Alexei Starobinsky for helpful discussions.
[1] J. D. Barrow and F. J. Tipler, Closed universes: their future evolution and final state, Mon. Not. R. Astron. Soc. 216, 395 (1985)

[2] A. Friedmann, Uber die Krummung des Raumes, Zeit. f. Physik 10, 377 (1922); Gen. Rel. Grav. 31, 1991 (1999)

[3] A. A. Starobinsky, Future and origin of our universe: Modern view, Grav. Cosmol. 6, 157-163 (2000)

[4] R. R. Caldwell, A phantom menace? Cosmological consequences of a dark energy component with super-negative equation of state, Phys. Lett. B 545, 23-29 (2002)

[5] R. R. Caldwell, M. Kamionkowski and N. N. Weinberg, Phantom energy: Dark energy with $w<-1$ causes a cosmic doomsday, Phys. Rev. Lett. 91, 071301 (2003)

[6] J. D. Barrow, G. J. Galloway and F. J. Tipler, The closeduniverse recollapse conjecture, Mon. Not. Roy. Astron. Soc. 223, 835-844 (1986)

[7] G. F. R. Ellis, Relativistic Cosmology, in General Relativity and Cosmology, Proc. Int. School of Phys. Enrico Fermi, Varenna Lectures vol. XLVII, ed. R. K. Sachs, Academic Press, New York, (1969), pp. 104-182; Gen.
Rel. Grav. 41, 581-660 (2009)

[8] J. D. Barrow, Sudden future singularities, Class. Quantum Grav. 21, L79-L82 (2004)

[9] J. D. Barrow, More general sudden singularities, Class. Quantum Grav. 21, 5619-5622 (2004)

[10] Y. Shtanov and V. Sahni, New cosmological singularities in braneworld models, Class. Quantum Grav. 19, L101L107 (2002)

[11] P. Tretyakov, A. Toporensky, Y. Shtanov and V. Sahni, Quantum effects, soft singularities and the fate of the universe in a braneworld cosmology, Class. Quantum Grav. 23, 3259-3274 (2006)

[12] J. D. Barrow and C. G. Tsagas, New isotropic and anisotropic sudden singularities, Class. Quantum Grav. 22, 1563-1571 (2005)

[13] J. D. Barrow and S. Z. W. Lip, Classical stability of sudden and big rip singularities, Phys. Rev. D 80, 043518 (2009)

[14] J. D. Barrow, S. Cotsakis and A. Tsokaros, A general sudden cosmological singularity, Class. Quantum Grav. 
27, 165017 (2010)

[15] M. P. Dạbrowski, Inhomogenized sudden future singularities, Phys. Rev. D 71, 103505 (2005)

[16] A. Balcerzak and M. P. Da̧browski, Strings at future singularities, Phys. Rev. D 73, 101301(R) (2006)

[17] C. Cattoën and M. Visser, Necessary and sufficient conditions for big bangs, bounces, crunches, rips, sudden singularities and extremality events, Class. Quantum Grav. 22, 4913 (2005)

[18] S. Nojiri, S. D. Odintsov and S. Tsujikawa, Properties of singularities in the (phantom) dark energy universe, Phys. Rev. D 71, 063004 (2005)

[19] J. D. Barrow, A. B. Batista, J. C. Fabris and S. Houndjo, Quantum particle production at sudden singularities, Phys. Rev. D 78, 123508 (2008); J. D. Barrow, A. B. Batista, J. C. Fabris, M. J. S. Houndjo and G. Dito, Sudden singularities survive massive quantum particle production, Phys. Rev. D 84, 123518 (2011); J. de Haro, J. Amoros, and E. Elizalde, Sudden singularities in semiclassical gravity, Phys. Rev. D 85, 123527 (2012); A. Yu. Kamenshchik, Quantum cosmology and late-time singularities, Class. Quantum Grav. 30, 173001 (2013)

[20] S. Nojiri and S. D. Odintsov, Quantum escape of sudden future singularity, Phys. Lett. B 595, 1-8 (2004); S. Nojiri and S. D. Odintsov, Final state and thermodynamics of a dark energy universe, Phys. Rev. D 70, 103522 (2004)

[21] K. Lake, Sudden future singularities in FLRW cosmologies, Class. Quantum Grav. 21, L129-L132 (2004)

[22] F. J. Tipler, Singularities in conformally flat spacetimes, Phys. Lett. A 64, 8-10 (1977)

[23] A. Krolak, Towards the proof of the cosmic censorship hypothesis, Class. Quantum Grav. 3, 267-280 (1986)

[24] L. Fernández-Jambrina and R. Lazkoz, Geodesic behavior of sudden future singularities, Phys. Rev. D 70, 121503(R) (2004); L. Fernández-Jambrina and R. Lazkoz, Classification of cosmological milestones, Phys. Rev. D 74, 064030 (2006)

[25] J. D. Barrow and S. Cotsakis, Geodesics at sudden singularities, Phys. Rev. D 88, 067301 (2013)

[26] V. Gorini, A. Kamenshchik, U. Moschella and V. Pasquier, Tachyons, scalar fields and cosmology, Phys. Rev. D 69, 123512 (2004)

[27] F. Cannata, A. Y. Kamenshchik and D. Regoli, Scalar field cosmological models with finite scale factor singularities, Phys. Lett. B 670, 241-245 (2009)

[28] L. Fernández-Jambrina, w-cosmological singularities, Phys. Rev. D 82, 124004 (2010)
[29] S. Appleby, R. Battye and A. Starobinsky, Curing singularities in cosmological evolution of $\mathrm{F}(\mathrm{R})$ gravity, JCAP 1006, 005 (2010)

[30] S. Myrzakul, R. Myrzakulov and L. Sebastiani, Inhomogeneous viscous fluids in FRW universe and finite-future time singularities, Astrophys. Space Sci. 350, 845-853 (2014)

[31] A. D. Rendall, Theorems on existence and global dynamics for the Einstein equations Living Rev. Relativity 8,6 (2005)

[32] A. D. Linde, Chaotic inflation, Phys. Lett. B 129, 177181 (1983)

[33] N. Kaloper and A. Padilla, The End, Phys. Rev. Lett. 114, 101302 (2015)

[34] G. F. R. Ellis and B. Schmidt, Singular space-times, Gen. Rel. Grav. 8, 915-953 (1977); G. F. R. Ellis and B. Schmidt, Classification of singular space-times, 10, 989-997 (1979)

[35] A. A. Coley, S. Hervik, W. C. Lim and M. A. H. MacCallum, Properties of kinematic singularities, Class. Quantum Grav. 26, 215008 (2009)

[36] U. Richard, Su un'equazione non lineare del secondo ordine, Rend. Sem. Math. Univ. Politec. Torino 10, 305324 (1951)

[37] K. Harigaya, M. Ibe, K. Schmitz and T. T. Yanagida, Chaotic inflation with a fractional power-law potential in strongly coupled gauge theories, Phys. Lett. B 720, 125129 (2013) and Dynamical fractional chaotic inflation, Phys. Rev. D 90, 123524 (2014)

[38] E. Silverstein and A. Westphal, Monodromy in the CMB: Gravity waves and string inflation, Phys. Rev. D 78, 106003 (2008)

[39] P. A. R. Ade et al, Planck 2013 results. XXII. Constraints on inflation Astron. Astrophys. 571, A22 (2014)

[40] D. N. Page, A fractal set of perpetually bouncing universes?, Class.Quantum Grav. 1, 417-427 (1984)

[41] Z. Keresztes, L. Á. Gergely and A. Yu. Kamenshchik, Paradox of soft singularity crossing and its resolution by distributional cosmological quantities, Phys. Rev. D 86, 063522 (2012)

[42] Z. Keresztes, L. Á. Gergely, A. Yu. Kamenshchik, V. Gorini, and D. Polarski, Soft singularity crossing and transformation of matter properties, Phys. Rev. D 88, 023535 (2013)

[43] L. Kofman, A. Linde and A. A. Starobinsky, Reheating after inflation, Phys. Rev. Lett. 73, 3195-3198 (1994) 